\title{
TABLE ANALYTIQLE
}

Exemples de présentation des références :

Sadler 133-135 : toute la communication de D. H. Sadler figurant aux pages i 33 à $\mathrm{s} 35$ incluses.

Vicente 109-111, 36 : partie de la communication de IR. O. Vicente couvrant toute la page 109 et se terminant à la ligne 36 de la page i 1 .

Fricke 11, 32-12, 29 : partie de la communication de W. Fricke allant de la ligne 32 , page i i à la ligne 29, p. 12 .

Procès-verbal 314, $4-28$ : lignes $4 \dot{a} 28$ de la page $31 / 4$ des procès-verbaux.

\section{I. - Constantes relatives à la Terre :}

$1^{\circ}$ Potentiel terrestre :

a. Constante de la gravitation géocentrique :

Kaula 24, 20-26, 3o; Cook 36, 7-24; 39, г7-40, $14 ; 43,36-44,3 ; 49,28-50,5$; Herrick 95-103; Procès-verbal 302, ı $6-26 ;$ 308, 9-30.

b. Gravité à la surface de la Terre :

Kaula 23, 26-24, 19 ; Cook 37, 10-38, $6 ; 42,3-17 ; 46,3\}-48,34 ; 57,19-59,7$; Procès-verbal 299, 3-300, $6 ;$ 307, 26-308, 23.

c. Coefficient d'ellipticité géopotentielle $\mathrm{J}_{2}$ :

Kaula 23, 13-19; Cook 37, 33-36; 39, 17-40, 1/4; 44, 36-45, 36; Jeffreys 70, 8-38; Böhme-Fricke 275-276, 25.

d. Autres coefficients du développement du potentiel terrestre:

Kaula 22, I-22; 26, 3i-27, 27; Cook 35, 4-36, 6 ; 44, 36-45, 36; Jeffreys 67, 29-71, 24; Brouwer 242, 23-244, п; Böhme-Fricke 274-276, 25; Procès-verbal 301, 7-1 2, 312, I3-18.

20 Forme de la Terre :

a. Géodésie :

Kaula 27, 28-30, 3; Cook 42, I 8-43, 9 .

b. Rayon terrestre :

Kaula 22, 23-23, 5; Cook 42, 18-43, 2; 48, 35-49-4; 57, 19-59, 7; Jeffreys 71, 25-73, 22 ; 75, 40-76, 5; Böhme-Fricke 273, 23-274; Procès-verbal 300, 8-34.

c. Applatissement :

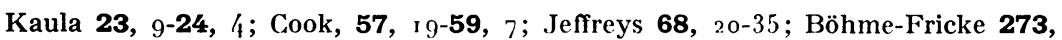
23-274.

Symposium U. A. I., no 21. 
3o Système GÉodésique :

Fricke 7, 33-8, 7; Kaula 23, 6-24, 19; Cook 44, 4-35; 57, 19-59, 7; Jeffreys 67, 19-28.

\section{II. - Constantes relatives au système Terre-Lune :}

I $^{\circ}$ Distance Terre-Lune et parallaxe lunaire :

Kaula 24, 28-25, I 8; Cook 40, I 5-41, I 5; 45, 37-46, 7; Jeffreys 75, 26-39; Yapplee et al. 82-92, Procès-verbal 301, r $6-25 ; 310,22-34$.

$2^{\circ}$ INÉgalité Lunaire :

Jeffreys 73, 23-74, 26 ; Vicente 111, 37-112, 17 ; Brouwer 246, 30-248; BöhmeFricke 279, 6-280, 28.

30 Masse de la Lune :

Kaula 25, 3-1 3; Cook 41, 16-42, 2; 46, 8-33; Jeffreys 75, 19-25; Vicente 111, $37-112,20$; Procès-verbal 302, 27-37; 311, 1/4-19.

$4^{\circ}$ Sélénodésie et figure de la lune :

Kaula 24, 28-25, 3; Cook 43, г 0-1 3; 49, 5-24; Jeffreys 76, 6-78, 3; Procès-verbal 302, 38-303, 5; 310, 3o-311, 4; 318, $18-37$; Résolution no $8322,1-9$; 324, $1-8$.

50 Occultations d'Étoiles par la Lune :

Cook 43, I 4-35; 49, 25-27; 59, 9-64; Yapplee et al. 91, 38-92, 7 .

\section{III. - Constantes relatives au système solaire :}

$I^{\circ}$ Parallaxe solaire et longueur de l'unité astronomique :

a. Méthodes optiques :

Fricke 12, 34-13, 9; 13, 28-15, 9; 15, 23-16, 17 ; Jeffreys 74, 27-75, 18 ; BöhmeFricke 276, 26-279, 5; Procès-verbal 307, 19-24; 311, 5-1 4; 311, 28-312, 4.

b. Méthodes radioélectriques :

Fricke 12, 3o-33; 13, 10-27; Muhleman 155, 18-169, 21 ; 170, 13-173, 11 ; Shapiro 178-215; Ponsonby et al. 217-218 ; Böhme-Fricke 276, 26-279, 5; Procèsverbal 305, 21-40; 307, 4-18; 311, 20-27; 319, 15-23; Résolution no 7 321, 36-39; 323, $32-35$.

c. Vitesse de la lumière :

Muhleman 154, 1 1-155, 17; Böhme-Fricke 271, 9-12.

d. Compatibilité des deux méthodes :

Fricke 15, 10-15, 22 ; Rabe 219-223 : Marsden 225-236 : Rabe 237-239 ; Procèsverbal 306-307, 3; 314, 29-40; Résolution no 6 321, 3o-35; 323, 26-3 I.

$2^{\circ}$ Théorie et masse des planètes :

Fricke 11, 32-12, 29; Herrick 105-108; Dùncombe 137; Kulikov 140-149; Muhleman 157, $10-158,18 ; 169,22-171,23$; Shapiro 202, 5-204; Rabe 219-223; Marsden 234, 14-236, I6; Rabe 237-239 ; Böhme-Fricke 280, 29-282, 14 ; Procèsverbal 303, $25-3 \mathrm{I} ; \mathbf{3 0 5}, 14-20 ; \mathbf{3 1 9}, 28-33$. 


\section{IV. - Les axes fondamentaux :}

Io Constante de la précession :

Fricke 17, 40-19; Vicente 111, г5-36; Boshniakovich 119-122; Böhme-Fricke 285-288; Procès-verbal 303, 7-16;314, 4-28.

$2^{\circ}$ Constante de la nutation :

Fricke 11, 18-27; Jefireys 78, 3-42; Vicente 109-111, 36; Fedorov 115-118; Böhme-Fricke 283, 22-284; Procès-verbal 302, $1-15$; 314, 4-28.

3० Constante de l'aberration :

Fricke 11, 28-3r; 16, 18-17, 39 ; Guinot 125-129; Potter 131-132; BöhmeFricke 282, I5-283, 21; Procès-verbal 303, 32-305, 13.

$4^{\circ}$ Divers :

a. Catalogues fondamentaux :

Fricke 9-11, 18; Boshniakovich 119-122; Procès-verbal 311, 35-312, 4.

b. Temps :

Fricke 8, 17-42; Sadler 133-135; Procès-verbal 303, г6-2/.

c. Variations des latitudes :

Jeffreys 78, 2-42; Fedorov 117, 1 0-118, 3.

\section{V. - Système de constantes astronomiques :}

Io Système existant :

Fricke 6-7, 32; Gook 34-35, 3; Herrick 96, 25-97, 30; 103-108; Wilkins 267, 3-268.

$2^{\circ}$ Relations entre les constantes (Voir aussi à chaque constante) :

Cook 38, 7-44, 3; 50, 6-51; 55-59, 7; Herrick 98, 4-101, 6; Brouwer 242-248; Wilkins 254, 3-256, 7; 256, 28-259 ; 260, 2ধ-263, г 7; Böhme-Fricke 271, г 3-273, 32,

$3 \circ$ Propositions concernant un nouveau système :

Cook 36, 25-37, 9; 44, 4-35; 52-54; Herrick 95-96, 4; 101, 33-103; Vicente 112, 2 1-113; Guinot 128, 3o-39; Muhleman 173, г 2-174; Wilkins 252-268; Procèsverbal 300, 7-34; 309-310, 11 ; 312, $3_{1}-314,2$; 314, 4-28; 317, $16-318,17$; Résolutions nos $1,2,3,4,5: 320,9-321,30 ; 322$, г $0-323,26$.

\section{VI. - Recommandations et programmes :}

Procès-verbal 296, 3o-298, 7 ; 299, 3-300, 6; 300, 3o-34; 312, 25-313, 7; 315, 17-317, 15 ; 318, 16-320, 5; Résolutions 320, 9-324. 